\title{
A NOTE ON THE HOMOLOGY OF FREE ABELIANIZED EXTENSIONS
}

\author{
BRIAN HARTLEY AND RALPH STÖHR
}

(Communicated by Warren J. Wong)

\begin{abstract}
Let $G$ be a group given by a free presentation $G=F / N$, and $N^{\prime}$ the commutator subgroup of $N$. The quotient $F / N^{\prime}$ is called a free abelianized extension of $G$. We study the integral homology of $F / N^{\prime}$. In particular, if $G$ has no elements of order $p$ ( $p$ an odd prime), we determine the $p$-torsion in dimension $p^{2}$ in terms of the modulo $p$ homology of $G$. This extends results of Kuz' $\min [5,6]$ describing the $p$-torsion in smaller dimensions. Our approach is based on examining the homology of $G$ with coefficients in symmetric powers of the augmentation ideal, which we relate to the integral homology of $F / N^{\prime}$.
\end{abstract}

\section{INTRODUCTION}

Let $G$ be a group and

$$
1 \rightarrow N \rightarrow F \rightarrow G \rightarrow 1
$$

a free presentation of $G$, in which $F$ is free and noncyclic. Let $\Phi=F / N^{\prime}$, and let $M=N / N^{\prime}$ be the relation module of $G$ coming from (1.1). The group $\Phi$ is called a free abelianized extension of $G$, and we have the exact sequence

$$
1 \rightarrow M \rightarrow \Phi \rightarrow G \rightarrow 1 \text {. }
$$

In his fundamental paper [5], Kuz' min has studied the integral homology groups $H_{n} \Phi$, and some additional information has been obtained in his recent paper [6]. Since the case $n=1$ is trivial $\left(H_{1} \Phi=F / F^{\prime}\right)$, the case $n=2$ is relatively easy and well understood (Hopf's formula gives $H_{2} \Phi \cong H_{0}(G, M \wedge M)$, and a description of the latter can be found in $[5,7])$, we focus on $n \geq 3$. For an abelian group $X$, we denote by $t X$ its torsion subgroup and, if $m$ is a natural number, we write $t_{m} X$ for the subgroup of all elements $x \in X$ with $m^{r} x=0$ for some $r \geq 1$. Kuz'min has proved that the torsion subgroup $t H_{n} \Phi$ is of finite exponent [5, Theorem 4], and pointed out that the exponent of the odd part of $t H_{n} \Phi$ divides $n$ [6, Theorem 1]. In the case when $G$ has no $p$-torsion ( $p$

Received by the editors May 29, 1990.

1980 Mathematics Subject Classification (1985 Revision). Primary 20J05, $20 \mathrm{E} 22$.

Key words and phrases. Homology of groups, free abelianized extensions, symmetric powers of modules. 
an odd prime), he established an isomorphism $t_{p} H_{p} G \cong H_{p+2}\left(G, \mathbb{Z}_{p}\right)$, where $\mathbb{Z}_{p}=\mathbb{Z} / p \mathbb{Z}$ regarded as a trivial $G$-module [5, Theorem 8]. In [6], Kuz'min has made the following conjectures on $p$-torsion $(p \geq 3)$ in $H_{n} \Phi$.

C1. For all $n \geq 3, p t_{p} H_{n} \Phi=0$.

C2. Suppose that $G$ has no $p$-torsion and let $s \geq 1$. Then

$$
t_{p} H_{p^{s}} \Phi \cong \bigoplus_{i=0}^{s-1} H_{p^{s}+2 p^{i}}\left(G, \mathbb{Z}_{p}\right)
$$

The results described above confirm $\mathrm{C} 1$ for all $n$ with $(n, p)=p$ or $(n, p)=$ 1 (in the latter case there is no $p$-torsion at all), and C2 for $s=1$. Also, Kuz' $\min$ announced without proof that

$$
t_{p} H_{m p} \Phi \cong H_{(p+2) m}\left(G, \mathbb{Z}_{p}\right)
$$

if $1 \leq m<p$ and $G$ has no $p$-torsion [6, p. 859].

In this paper we shall prove (1.2) for the case $s=2$, and obtain some information on the general case. Our method will also establish (1.3).

For an abelian group $X$, let $\bigwedge^{n} X$ denote the $n$th exterior power of $X$ and $X^{n}$ the $n$th symmetric power (our notation differs here from Kuz'min's). If $X$ is a $G$-module, then these are $G$-modules via the diagonal action. Kuz'min's approach involves showing that

$$
t H_{n} \Phi \cong t_{n} H_{0}\left(G, \bigwedge^{n} M\right)
$$

"up to 2-torsion," meaning that the odd parts of these groups are isomorphic [5, Theorem 7]. Because of (1.4), questions about odd torsion in $H_{n} \Phi$ are equivalent to questions about homology of $G$ with coefficients in exterior powers of $M$, which was extensively examined in $[5,6]$. An alternative treatment of $H_{*}\left(G, \Lambda^{n} M\right)$ has been given by Hannebauer and the second author [3]. In particular, they improved an "up to 2-torsion" result of [6] by showing that $H_{k}\left(G, \Lambda^{n} M\right)(k \geq 1)$ and $t H_{0}\left(G, \Lambda^{n} M\right)$ have exponent dividing $2 n(n-1)$ for all $n \geq 2$.

Our approach is to transform the problem to one about homology of $G$ with coefficients in symmetric powers $(I G)^{n}$ of the augmentation ideal $I G$ of $\mathbb{Z} G$, using the exact sequence

$$
\begin{aligned}
0 \rightarrow \bigwedge^{n} M \rightarrow \bigwedge^{n-1} M \otimes P \rightarrow \cdots & \rightarrow \bigwedge^{n-i} M \otimes P^{i} \rightarrow \cdots \\
& \rightarrow M \otimes P^{n-1} \rightarrow P^{n} \rightarrow(I G)^{n} \rightarrow 0,
\end{aligned}
$$

which comes from the relation sequence

$$
0 \rightarrow M \rightarrow P \rightarrow I G \rightarrow 0
$$

and $\left[1, \S 9\right.$, ex. 2]. In the sequence (1.6), $P$ is the free $G$-module $I F \otimes_{G} \mathbb{Z} G$. If $G$ has no $n$ !-torsion, then the modules $P^{i}(1 \leq i \leq n)$ are $G$-free [4, Lemma 
4.1], and we can use dimension shifting through (1.5) to relate the homologies of $G$ with coefficients in $\wedge^{n} M$ and $(I G)^{n}$. With a little more argument we get (see $\S 2)$

Proposition 1.1. If $G$ has no p-torsion, then

$$
t_{p} H_{k}\left(G, \bigwedge^{n} M\right) \cong t_{p} H_{k+n}\left(G,(I G)^{n}\right)
$$

for all $k \geq 0, n \geq 1$.

Our main results refer to $H_{*}\left(G,(I G)^{n}\right)$ for prime powers $n=p^{s}$, including $p=2$.

Theorem 1. Let $p$ be a prime and suppose that $G$ has no p-torsion. Then, for all $k \geq 0$,

$$
t_{p} H_{k}\left(G,(I G)^{p^{2}}\right) \cong H_{k+2}\left(G, \mathbb{Z}_{p}\right) \oplus H_{k+2 p}\left(G, \mathbb{Z}_{p}\right) .
$$

Theorem 2. Let $p$ be a prime and suppose that $G$ has no p-torsion. Then, for all $k \geq 0$ and $s \geq 1$,

$$
t_{p} H_{k}\left(G,(I G)^{p^{s}}\right) \cong H_{k+2}\left(G, \mathbb{Z}_{p}\right) \oplus Y,
$$

where $p^{s-1} Y=0$.

We mention that, for any group $G$ and any $n \geq 2$, the groups $H_{k}\left(G,(I G)^{n}\right)$ $(k \geq 1)$ and $t H_{0}\left(G,(I G)^{n}\right)$ are annihilated by $2 n(n-1)$ [4, Theorem 5.1], so that $t_{p} H_{k}\left(G,(I G)^{n}\right)=0$ for all $n \not \equiv 0,1 \bmod p$. Now, if $p$ is an odd prime, our theorems together with Proposition 1.1 and (1.4) imply immediately the following

Corollary 3. Let $p$ be an odd prime and suppose that $G$ has no p-torsion. Then

$$
t_{p} H_{p^{2}} \Phi \cong H_{p^{2}+2}\left(G, \mathbb{Z}_{p}\right) \oplus H_{p^{2}+2 p}\left(G, \mathbb{Z}_{p}\right),
$$

and, for all $s \geq 1$,

$$
t_{p} H_{p^{s}} \Phi \cong H_{p^{s}+2}\left(G, \mathbb{Z}_{p}\right) \oplus Y
$$

where $p^{s-1} Y=0$.

Symmetric powers of $I G$ will be studied by embedding them in symmetric powers of $\mathbb{Z} G$ and using the $G$-module maps $\tau(n):(\mathbb{Z} G)^{n} \rightarrow(\mathbb{Z} G)^{n-1}$ given by

$$
\left(g_{1} \circ g_{2} \circ \cdots \circ g_{n}\right) \tau(n)=\sum_{i=1}^{n} g_{1} \circ \cdots \circ \hat{g}_{i} \circ \cdots \circ g_{n},
$$

where $g_{1}, \ldots, g_{n} \in G$ and the circumflex denotes that $g_{i}$ is omitted. The kernel of $\tau(n)$ is $(I G)^{n}$ (see $\S 2$ ). These maps make sense also when $\mathbb{Z}$ is replaced by any commutative ring with 1 , and will not be distinguished notationally. We remark in passing that if $\bigoplus_{n=0}^{\infty}(\mathbb{Z} G)^{n}$, the symmetric algebra on $\mathbb{Z} G$ (with $\left.(\mathbb{Z} G)^{0}=\mathbb{Z}\right)$, is regarded as the polynomial ring on the group elements as indeterminates, then $\bigoplus_{n=0}^{\infty} \tau(n)$ is the sum of the first partial derivatives with respect to the group elements. 
An interesting property of these maps that emerges during our discussion is the following.

Proposition 1.2. Let $p$ be a prime. Then

$$
\begin{aligned}
\cdots \rightarrow\left(\mathbb{Z}_{p} G\right)^{r p} \rightarrow\left(\mathbb{Z}_{p} G\right)^{r p-1} \rightarrow\left(\mathbb{Z}_{p} G\right)^{(r-1) p} \rightarrow\left(\mathbb{Z}_{p} G\right)^{(r-1) p-1} & \rightarrow \cdots \\
\rightarrow\left(\mathbb{Z}_{p} G\right)^{p} \rightarrow\left(\mathbb{Z}_{p} G\right)^{p-1} & \rightarrow \mathbb{Z}_{p} \rightarrow 0,
\end{aligned}
$$

where $\left(\mathbb{Z}_{p} G\right)^{r p} \rightarrow\left(\mathbb{Z}_{p} G\right)^{r p-1}$ is $\tau(r p) \quad(r=1,2, \ldots)$, and $\left(\mathbb{Z}_{p} G\right)^{r p-1} \rightarrow$ $\left(\mathbb{Z}_{p} G\right)^{(r-1) p}$ is $\tau(r p-1) \tau(r p-2) \cdots \tau((r-1) p+1)(r=1,2, \ldots)$, is a $\mathbb{Z}_{p} G$ resolution of $\mathbb{Z}_{p}$.

If $G$ is torsion-free, the modules $\left(\mathbb{Z}_{p} G\right)^{n}$ are free $\mathbb{Z}_{p} G$-modules [4, Lemma 4.1] and (1.7) is a free $\mathbb{Z}_{p} G$-resolution of $\mathbb{Z}_{p}$. If $G$ is merely $p$-torsion free, the symmetric powers of $\mathbb{Z}_{p} G$ need not be free, but their homology is concentrated in dimension zero (see $\S 2$ ).

Some of this work was carried out while the first author was visiting the KarlWeierstraß-Institut für Mathematik, Berlin, under the auspices of the exchange agreement between the Royal Society of London and the Akademie der Wissenschaften der DDR. He thanks these bodies for their support and his hosts for their kind hospitality.

\section{SYMMETRIC POWERS OF GROUP RINGS AND FREE MODULES}

In this section, let $S$ be a commutative ring with 1 . We later use $R$ to denote the integers localized at a fixed prime $p$. Let the elements of $G$ be totally ordered in any way. Then $(S G)^{n}$ has an $S$-basis consisting of all elements

$$
g_{1} \circ g_{2} \circ \cdots \circ g_{n} \quad\left(g_{i} \in G, g_{1} \leq g_{2} \leq \cdots \leq g_{n}\right)
$$

By replacing each $g_{i} \neq 1$ by $1+\left(g_{i}-1\right)$ and expanding, we easily see that the elements

$\left(g_{1}-1\right) \circ \cdots \circ\left(g_{l}-1\right) \circ 1 \circ \cdots \circ 1 \quad\left(g_{i} \in G, g_{i} \neq 1, g_{1} \leq \cdots \leq g_{l}, 0 \leq l \leq n\right)$

also form an $S$-basis of $(S G)^{n}$, which we call the augmentation basis. In particular, the elements (2.1) with $l=n$ form an $S$-basis for the canonical image of $\left(I_{S} G\right)^{n}$, the $n$th symmetric power of the augmentation ideal $I_{S} G$ of $S G$, in $(S G)^{n}$.

Consider the $G$-module homomorphism $\tau(n):(S G)^{n} \rightarrow(S G)^{n-1}$ defined by

$$
\left(\alpha_{1} \circ \cdots \circ \alpha_{n}\right) \tau(n)=\sum_{i=1}^{n}\left(\alpha_{i} \varepsilon\right) \alpha_{1} \circ \cdots \circ \hat{\alpha}_{i} \circ \cdots \circ \alpha_{n},
$$

where $\alpha_{1}, \ldots, \alpha_{n} \in S G$ and $\varepsilon$ is the augmentation map $S G \rightarrow S$. This is clearly the $\tau(n)$ described in the introduction. On an element $(2.1), \tau(n)$ takes the very simple form

$$
\left(\left(g_{1}-1\right) \circ \cdots \circ\left(g_{l}-1\right) \circ 1 \circ \cdots \circ 1\right) \tau(n)=(n-l)\left(g_{1}-1\right) \circ \cdots \circ\left(g_{l}-1\right) \circ 1 \circ \cdots \circ 1
$$


for $l<n$, and

$$
\left(\left(g_{1}-1\right) \circ \cdots \circ\left(g_{n}-1\right)\right) \tau(n)=0
$$

for $l=n$. The number of ones in (2.2) is clear from the context. Note that the right-hand side of (2.2) is a scalar multiple of an element of the augmentation basis of $(S G)^{n-1}$.

It will be convenient to introduce the following notation. Let $\Gamma$ be the set of all sequences

$$
\gamma=\left(g_{1}, \ldots, g_{l}\right)
$$

of finite length $l(l=0,1,2, \ldots)$ with $g_{i} \in G, g_{i} \neq 1$ and $g_{1} \leq \cdots \leq g_{l}$, including the empty sequence $\gamma_{0}$ of length 0 . Let $\Gamma_{n} \subseteq \Gamma$ denote the subset of all $\gamma$ with length $\leq n$. To any $n \geq 0$ and any sequence (2.4) we assign an element $\xi(n, \gamma) \in(S G)^{n}$ by setting

$$
\xi(n, \gamma)=\left(g_{1}-1\right) \circ \cdots \circ\left(g_{l}-1\right) \circ 1 \circ \cdots \circ 1 \text { for } \gamma \in \Gamma_{n}
$$

(here we have $n-l$ ones; in particular, $\xi\left(n, \gamma_{0}\right)=1 \circ 1 \circ \cdots \circ 1$ ), and

$$
\xi(n, \gamma)=0 \quad \text { for } \gamma \in \Gamma \backslash \Gamma_{n} .
$$

For $n>m \geq 0$ let $\sigma(n, m)$ denote the homomorphism $(S G)^{n} \rightarrow(S G)^{m}$ defined as the composite $\sigma(n, m)=\tau(n) \tau(n-1) \cdots \tau(m+1)$. For an element (2.4) of $\Gamma$ and $n>m$ we put

$$
\kappa(n, m, \gamma)=(n-l)(n-l-1) \cdots(m+1-l)
$$

so $\kappa(n, m, \gamma) \in S$ is the product of the $n-m$ successive numbers from $m+1-l$ to $n-l$. Now we state some rather obvious properties of these notions.

Lemma 2.1. (i) For any $n \geq 0$, the augmentation basis of $(S G)^{n}$ consists of all elements $\xi(n, \gamma)$ with $\gamma \in \Gamma_{n}$.

(ii) For $n>m \geq 0$ and $\gamma \in \Gamma$ we have

$$
\xi(n, \gamma) \sigma(n, m)=\kappa(n, m, \gamma) \xi(m, \gamma) .
$$

(iii) If $\Omega$ is a finite subset of $\Gamma_{n}$, then a linear combination

$$
\sum_{\gamma \in \Omega} \alpha(\gamma) \xi(n, \gamma) \in(S G)^{n}
$$

$(\alpha(\gamma) \in S, \alpha(\gamma) \neq 0)$ is in the kernel of $\sigma(n, m)$ if and only if for all $\gamma \in \Omega$ either $\gamma \in \Gamma_{n} \backslash \Gamma_{m}$ or $\alpha(\gamma) \kappa(n, m, \gamma)=0$ in $S$.

(iv) If $S$ is a domain of characteristic zero, then the elements $\xi(n, \gamma)$ with $\gamma \in \Gamma_{n} \backslash \Gamma_{m}$ form an $S$-basis for the kernel of $\sigma(n, m)$. In particular, the kernel of $\tau(n)=\sigma(n, n-1)$ coincides with the canonical image of $\left(I_{S} G\right)^{n}$ in $(S G)^{n}$. Proof. (i) is clear from the definition. For $m=n-1 \quad(\sigma(n, n-1)=\tau(n))$, (2.5) is essentially the same as (2.2), (2.3). The case $m<n-1$ follows by 
trivial induction on $n-m$. Finally, (iii) and (iv) are obvious consequences of (i) and (ii).

Proposition 1.2 is now easy to establish.

Proof of Proposition 1.2. We work here with $S=\mathbb{Z}_{p}$. Let $n \geq 1$. In view of Lemma 2.1 (iii), the kernel of $\tau(n):\left(\mathbb{Z}_{p} G\right)^{n} \rightarrow\left(\mathbb{Z}_{p} G\right)^{n-1}$ has a $\mathbb{Z}_{p}$-basis consisting of all elements $\xi(n, \gamma)$ such that $\gamma \in \Gamma_{n}$ and $\kappa(n, n-1, \gamma) \equiv$ $0 \bmod p$ (on noting that $\gamma \in \Gamma_{n} \backslash \Gamma_{n-1}$ implies $\kappa(n, n-1, \gamma)=0$ ). Now

$$
\xi(n+p-1, \gamma) \sigma(n+p-1, n)=\kappa(n+p-1, n, \gamma) \xi(n, \gamma) \text {. }
$$

If $\gamma$ has length $l$, one has $\kappa(n, n-1, \gamma)=n-l$ and

$$
\kappa(n+p-1, n, \gamma)=(n+p-1-l)(n+p-2-l) \cdots(n+1-l) .
$$

Consequently, $\kappa(n, n-1, \gamma) \equiv 0 \bmod p \quad$ implies that $\kappa(n+p-1, n, \gamma)$ is a unit in $\mathbb{Z}_{p}$, and (2.6) gives $\operatorname{im} \sigma(n+p-1, n)=\operatorname{ker} \tau(n)$ for all $n \geq 1$. Similarly, we get $\operatorname{im} \tau(n+1)=\operatorname{ker} \sigma(n, n-p+1)$ for all $n \geq p-1$. In particular, (1.7) is exact.

The symmetric powers $\left(\mathbb{Z}_{p} G\right)^{n}(n \geq 1)$ are $\mathbb{Z}_{p} G$-free if $G$ is torsion-free [4, Lemma 4.1]. So we can state the following

Corollary 2.2. If $G$ is torsion-free, then (1.7) is a free $\mathbb{Z}_{p} G$-resolution of $\mathbb{Z}_{p}$.

In the sequel we will work with groups that are $p$-torsion free, that is, they have no elements of order $p$. For such groups we have

Lemma 2.3. Let $p$ be a prime, $B$ a free $S G$-module. Suppose that $G$ is $p$ torsion free and every prime $q \neq p$ is invertible in $S$. Then $H_{k}\left(G, D \otimes_{S} B^{n}\right)=0$ for all $S G$-modules $D, k \geq 1$ and $n \geq 1$.

Proof. The symmetric power $B^{n}$ is a permutation module and the stabilizers of all elements of its canonical permutation basis are finite subgroups of $G$ [4, $\S 4]$. Consequently, $B^{n}$ decomposes into the direct sum of induced modules

$$
\operatorname{Ind}_{H}^{G} S=S \otimes_{H} \quad H \leq G,|H|<\infty
$$

(see for example [2, III.5]). Therefore, to prove our lemma it suffices to show that $H_{k}\left(G, D \otimes_{S} \operatorname{Ind}_{H}^{G} S\right)=0$ for all $k \geq 1$ and finite $H \leq G$. Now

$$
D \otimes_{S} \operatorname{Ind}_{H}^{G} S \cong \operatorname{Ind}_{H}^{G}\left(\operatorname{Res}_{H}^{G} D \otimes_{S} S\right)
$$

By Shapiro's Lemma,

$$
H_{k}\left(G, D \otimes_{S} \operatorname{Ind}_{H}^{G} S\right) \cong H_{k}\left(H, \operatorname{Res}_{H}^{G} D\right) .
$$

The right-hand side is annihilated by $|H|$ if $k \geq 1$. The hypotheses imply that $|H|$ is invertible in $S$, whence the assertion.

Recall that $R$ denotes the integers localized at $p$, and that tensoring with $R$, that is, localizing at $p$, is an exact functor on the category of abelian groups. 
Thus $H_{k}(G, X \otimes R) \cong H_{k}(G, X) \otimes R$ for any $G$-module $X$. We deduce

Lemma 2.4. $t_{p} H_{k}(G, X) \cong t H_{k}(G, X \otimes R)$ for any G-module $X$.

For this reason, we usually work over $R$ instead of $\mathbb{Z}$ from now on.

Proof of Proposition 1.1. By Lemma 2.4 it suffices to show that

$$
t H_{k}\left(G, \bigwedge^{n}(M \otimes R)\right) \cong t H_{k+n}\left(G,\left(I_{R} G\right)^{n}\right) .
$$

Consider the exact sequence (1.5) localized at $p$. Then Lemma 2.3 tells us that all its terms except $\Lambda^{n}(M \otimes R)$ and $\left(I_{R} G\right)^{n}$ have zero homology in positive dimensions. So for $k \geq 1$, Proposition 1.1 follows by dimension shifting through (1.5) localized at $p$. For $k=0$, note that $\bigwedge^{n-1} M \otimes P$ is actually free. So, by dimension shifting we get

$$
\operatorname{ker}\left(H_{0}\left(G, \bigwedge^{n}(M \otimes R)\right) \rightarrow H_{0}\left(G, \bigwedge^{n-1} M \otimes P \otimes\right)\right) \cong H_{n}\left(G,\left(I_{R} G\right)^{n}\right) .
$$

The proposition follows on noting that $t H_{0}\left(G, \Lambda^{n}(M \otimes R)\right)$ is contained in the kernel on the left-hand side (indeed, the corresponding image lies in the free $R$-module $\left.H_{0}\left(G, \wedge^{n-1} M \otimes P \otimes R\right)\right)$.

\section{HOMOLOGY OF SYMMETRIC POWERS OF $I G$}

In this section we are concerned with discussing $p$-torsion in $H_{*}\left(G,(I G)^{n}\right)$, where $p$ is an arbitrary prime. We shall prove our two theorems and establish (1.3). By Lemma 2.4, there is an isomorphism

$$
t_{p} H_{k}\left(G,(I G)^{n}\right) \cong t H_{k}\left(G,(I G)^{n} \otimes R\right),
$$

so we can work with symmetric powers of $I_{R} G$, the augmentation ideal of the localized group ring $R G$. Throughout this section we will assume that $G$ is $p$-torsion free.

By Lemma 2.1(iv) we have a 4-term exact sequence

$$
0 \rightarrow\left(I_{R} G\right)^{n} \rightarrow(R G)^{n} \stackrel{\tau(n)}{\longrightarrow}(R G)^{n-1} \rightarrow \operatorname{coker} \tau(n) \rightarrow 0 .
$$

By Lemma 2.3, the homology of the middle terms is zero in positive dimensions, and we get by dimension shifting

$$
H_{k}\left(G,\left(I_{R} G\right)^{n}\right) \cong H_{k+2}(G, \operatorname{coker} \tau(n)) \quad(k \geq 1, n \geq 2) .
$$

If $n=p^{s}(s \geq 1)$, we also have

$$
t H_{0}\left(G,\left(I_{R} G\right)^{n}\right) \cong t H_{2}(G, \operatorname{coker} \tau(n)) .
$$

This can be proved along the same lines as the case $k=0$ in the proof of Proposition 1.1 on noting that $(R G)^{p^{s}}$ is free [4, Lemma 4.1]. Thus, we transfer our attention to $\operatorname{coker} \tau(n)$, the $R$-structure of which is quite transparent. Recall that $(R G)^{n-1}$ has an $R$-basis consisting of all elements $\xi(n-1, \gamma)$ with $\gamma \in \Gamma_{n-1}($ see $\S 2)$. Let $\pi(m)$ denote the largest power of $p$ dividing the natural 
number $m$. The following is clear from Lemma 2.1.

Lemma 3.1. The cokernel of $\tau(n)$ is a direct sum of finite cyclic p-groups generated by the elements $\xi(n-1, \gamma)+\operatorname{im} \tau(n), \gamma \in \Gamma_{n-1}$, such that $p$ divides $\kappa(n, n-1, \gamma)$. The order of $\xi(n-1, \gamma)+\operatorname{im} \tau(n)$ is $\pi(\kappa(n, n-1, \gamma))$.

Now consider the commutative diagram

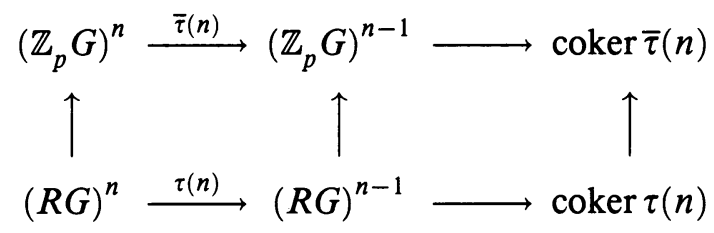

where the vertical maps are reduction $\bmod p$ and the notation $\bar{\tau}(n)$ is introduced to distinguish the appropriate versions of $\tau(n)$. If $n=r p \quad(r \geq 1)$, the homology of coker $\bar{\tau}(n)$ can be computed by dimension shifting through (1.7) (using Lemma 2.3), and in view of $\operatorname{coker} \tau(n) \otimes \mathbb{Z}_{p} \cong \operatorname{coker} \bar{\tau}(n)$ we get

$$
H_{k}\left(G, \operatorname{coker} \tau(r p) \otimes \mathbb{Z}_{p}\right) \cong H_{k+2(r-1)}\left(G, \mathbb{Z}_{p}\right) \quad(k \geq 1, r \geq 1) .
$$

If $r<p$, Lemma 3.1 tells us that coker $\tau(r p)$ is of exponent $p$. Hence

$$
\operatorname{coker} \tau(r p) \cong \operatorname{coker} \tau(r p) \otimes \mathbb{Z}_{p}
$$

and from (3.1), (3.2) and (3.4) we deduce

Proposition 3.2. Let $G$ be $p$-torsion free and $1 \leq r<p$. Then

$$
t_{p} H_{k}\left(G,(I G)^{r p}\right) \cong H_{k+2 r}\left(G, \mathbb{Z}_{p}\right) \quad(k \geq 1) .
$$

In view of (1.4) and Proposition 1.1 this gives

Corollary 3.3 (Kuz'min [6]). Suppose that $p$ is an odd prime, $G$ is p-torsion free and $1 \leq r<p$. Then $t_{p} H_{r p} \Phi \cong H_{(p+2) r}\left(G, \mathbb{Z}_{p}\right)$.

We conclude with the proofs of our two theorems. Since the case $n=p$ is established in Proposition 3.2 (for $r=1$ the assertion extends to $k \geq 0$; and previously in $[3,5]$ ), we can assume that $n=p^{s}$ with $s \geq 2$.

Proof of Theorem 2. Consider the commutative diagram

$$
\begin{aligned}
& (R G)^{p^{s}} \stackrel{\sigma\left(p^{s}, p^{s}-p^{s-1}\right)}{\longrightarrow}(R G)^{p^{s}-p^{s-1}} \longrightarrow \operatorname{coker} \sigma\left(p^{s}, p^{s}-p^{s-1}\right) \\
& \| \uparrow_{\sigma\left(p^{s}, p^{s}-p^{s-1}\right)} \uparrow_{\varphi} \\
& (R G)^{p^{s}} \longrightarrow \frac{\tau\left(p^{s}\right)}{\longrightarrow}(R G)^{p^{s}-1} \longrightarrow \quad \operatorname{coker} \tau\left(p^{s}\right)
\end{aligned}
$$

where $\varphi$ is the induced homomorphism. By Lemma 2.1 we have for all $\gamma \in \Gamma_{p^{s}}$,

$$
\xi\left(p^{s}, \gamma\right) \sigma\left(p^{s}, p^{s}-p^{s-1}\right)=\kappa\left(p^{s}, p^{s}-p^{s-1}, \gamma\right) \xi\left(p^{s}-p^{s-1}, \gamma\right),
$$

where $\kappa\left(p^{s}, p^{s}-p^{s-1}, \gamma\right)$ is a product of $p^{s-1}$ successive numbers $\leq p^{s}$ (and $\left.\geq-p^{s-1}\right)$. Let $t=\pi\left(p^{s-1} !\right)$. Then $\pi\left(\kappa\left(p^{s}, p^{s}-p^{s-1}, \dot{\gamma}_{0}\right)\right)=p^{t+1}$ and 
$\pi\left(\kappa\left(p^{s}, p^{s}-p^{s-1}, \gamma\right)\right)=p^{t}$ for all $\gamma \in \Gamma_{p^{s}-p^{s-1}} \backslash \gamma_{0}$. Hence, for the cokernel of $\sigma\left(p^{s}, p^{s}-p^{s-1}\right)$ there is an exact sequence

$$
0 \rightarrow \mathbb{Z}_{p} \rightarrow \operatorname{coker} \sigma\left(p^{s}, p^{s}-p^{s-1}\right) \rightarrow(R G)^{p^{s}-p^{s-1}} \otimes \mathbb{Z}_{p^{t}} \rightarrow 0,
$$

where the embedding of $\mathbb{Z}_{p}$ into the cokernel is given by

$$
1 \mapsto p^{t} \xi\left(p^{s}-p^{s-1}, \gamma_{0}\right)+\operatorname{im} \sigma\left(p^{s}, p^{s}-p^{s-1}\right) .
$$

By Lemma 3.1, $\operatorname{coker} \tau\left(p^{s}\right)$ is a direct sum of cyclic $p$-groups. Note that the order of $\xi\left(p^{s}-1, \gamma_{0}\right)+\operatorname{im} \tau\left(p^{s}\right)$ is $p^{s}$, whereas the orders of the remaining generators of coker $\tau\left(p^{s}\right)$ are $<p^{s}$. Hence, we have an exact sequence

$$
0 \rightarrow \mathbb{Z}_{p} \rightarrow \operatorname{coker} \tau\left(p^{s}\right) \rightarrow \operatorname{coker} \tau\left(p^{s}\right) \otimes \mathbb{Z}_{p^{s-1}} \rightarrow 0,
$$

where the embedding $\mathbb{Z}_{p} \rightarrow \operatorname{coker} \tau\left(p^{s}\right)$ is given by

$$
1 \mapsto p^{s-1} \xi\left(p^{s-1}-1, \gamma_{0}\right)+\operatorname{im} \tau\left(p^{s}\right) .
$$

Now consider (3.5). We have

$$
\begin{aligned}
p^{s-1} & \xi\left(p^{s}-1, \gamma_{0}\right) \sigma\left(p^{s}-1, p^{s}-p^{s-1}\right) \\
& =p^{s-1} \kappa\left(p^{s}-1, p^{s}-p^{s-1}\right) \xi\left(p^{s}-p^{s-1}, \gamma_{0}\right) \\
& =p^{t} u \xi\left(p^{s}-p^{s-1}, \gamma_{0}\right),
\end{aligned}
$$

where $u$ is a unit in $R$. Hence the induced homomorphism $\varphi$ maps $p^{s-1} \times$ coker $\tau\left(p^{s}\right)\left(\cong \mathbb{Z}_{p}\right)$ isomorphically onto $p^{t} \operatorname{coker} \sigma\left(p^{s}, p^{s}-p^{s-1}\right)\left(\cong \mathbb{Z}_{p}\right)$, so $\varphi$ induces a homomorphism of exact sequences

$$
\begin{array}{ccc}
0 \longrightarrow \mathbb{Z}_{p} & \longrightarrow \operatorname{coker} \sigma\left(p^{s}, p^{s}-p^{s-1}\right) & \longrightarrow \\
& \uparrow & (R G)^{p^{s}-p^{s-1}} \otimes \mathbb{Z}_{p^{t}} \longrightarrow 0 \\
0 \longrightarrow \mathbb{Z}_{p} \longrightarrow \operatorname{coker} \tau\left(p^{s}\right) & \longrightarrow \operatorname{coker} \tau\left(p^{s}\right) \otimes \mathbb{Z}_{p^{s-1}} \longrightarrow 0
\end{array}
$$

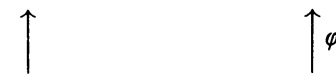

where the first vertical arrow is an isomorphism. By applying the homology functor we get the commutative diagram

$$
\begin{aligned}
& 0 \rightarrow H_{k}\left(G, \mathbb{Z}_{p}\right) \rightarrow H_{k}\left(G, \operatorname{coker} \sigma\left(p^{s}, p^{s}-p^{s-1}\right)\right) \rightarrow 0 \\
& \uparrow \rightarrow H_{k}\left(G, \mathbb{Z}_{p}\right) \rightarrow H_{k}\left(G, \operatorname{coker} \tau\left(p^{s}\right)\right) \rightarrow H_{k}\left(G, \operatorname{coker} \tau\left(p^{s}\right) \otimes \mathbb{Z}_{p^{s-1}}\right) \longrightarrow H_{k-1}\left(G, \mathbb{Z}_{p}\right) \longrightarrow \cdots
\end{aligned}
$$

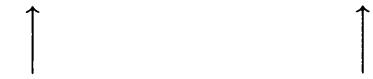

in which $H_{k}\left(G, \mathbb{Z}_{p}\right) \rightarrow H_{k}\left(G, \mathbb{Z}_{p}\right)$ is an isomorphism. This yields

$$
H_{k}\left(G, \operatorname{coker} \tau\left(p^{s}\right)\right) \cong H_{k}\left(G, \mathbb{Z}_{p}\right) \oplus H_{k}\left(G, \operatorname{coker} \tau\left(p^{s}\right) \otimes \mathbb{Z}_{p^{s-1}}\right)
$$

for all $k \geq 0$. Since $H_{k}\left(G, \operatorname{coker} \tau\left(p^{s}\right) \otimes \mathbb{Z}_{p^{s-1}}\right)$ is annihilated by $p^{s-1}$, Theorem 2 follows in view of (3.1), (3.2) and (3.3).

Proof of Theorem 1. Here we have $s=2$ and the theorem follows by (3.6) and (3.4) with $r=p$. 


\section{REFERENCES}

1. N. Bourbaki, Algèbre, Chapitre X: Algèbre homologique, Springer-Verlag, Paris, New York, Barcelona, and Milan, 1980.

2. K. S. Brown, Cohomology of groups, Springer-Verlag, New York, Heidelberg, and Berlin, 1982.

3. Torsten Hannebauer and Ralph Stöhr, Homology of groups with coefficients in free metabelian Lie powers and exterior powers of relation modules and applications to group theory, Proc. Second Internat. Group Theory Conf. Bressanone/Brixen 1989, Rend. Circ. Mat. Palermo (2) Suppl. 23 (1990), 77-113.

4. B. Hartley and R. Stöhr, Homology of higher relation modules and torsion in free central extensions of groups, Proc. London Math. Soc. (3) 62 (1991), 325-352.

5. Yu. V. Kuz' min, Homology theory of free abelianized extensions, Comm. Algebra 16 (1988), 2447-2533.

6. _ On some properties of free abelianized extensions, Mat. Sb. (N.S.) 180 (1989), 850-862. (Russian)

7. R. Stöhr, On elements of order four in certain free central extensions of groups, Math. Proc. Cambridge Philos. Soc. 106 (1989), 13-28.

Department of Mathematics, University of Manchester, Manchester M13 9PL, EngLAND

Department of Mathematics, UMiST, PO Box 88, Manchester M60 1QD, England 